\title{
Postoperative Quality of Recovery (QoR) after Ambulatory Laparoscopic Cholecystectomy in Patients with Deep Neuromuscular Block. A Prospective Cohort Study.
}

\author{
Martínez Giner J, Burchés E, Reyes A, Rodríguez M, Velasco E, Agustí S, López Gil M, Zaballos M \\ HOSPITAL GENERAL UNIVERSITARIO GREGORIO MARAÑÓN
}

\section{Background and Goal of study}

Laparoscopic cholecystectomy (LC) is one of the most common procedures performed in the world. Deep neuromuscular blockade (NMB) and low-pressure pneumoperitoneum, has recently been shown to improve surgical working conditions associated with a positive effect in recovery, reducing postoperative pain. Most studies evaluating recovery after LC, have ignored quality of recovery ( $Q \circ R)$ from the patient's perspective. We aimed to evaluate the QoR of patients undergoing $C L$ in whom deep NMB was performed as part of the anesthetic management.

\section{Material and Methods}

After IRB approval 75 patients scheduled for CL (ASA IIII), $51 \pm 14$ years old were enrolled. The anaesthetic protocol included multimodal analgesia (NSAIDs, wound infiltration with local anaesthetics, low doses of fentanyl), prophylaxis of postoperative nausea and vomiting and dexamethasone. Deep NMB was instituted during pneumoperitoneum (posttetanic count (PTC) of 1 or 2) and was maintained until the end of the surgery. The NMB was reversed at the end of the intervention with sugammadex at a dose of $4 \mathrm{mg} / \mathrm{kg}$, confirming the adequate recovery before extubation. QoR-15 recovery patient survey was performed, before and $24 \mathrm{~h}$ after surgery, with a maximum score of 150 .

\begin{tabular}{|l|}
\hline \multicolumn{1}{|c|}{ Quality of Recovery 15 Patient Survey } \\
\hline PART A: \\
How have you been feeling in the last 24 hours? \\
\hline 1. Able to breathe easily \\
2. Been able to enjoy food \\
3. Feeling rested \\
4. Have had a good sleep \\
5. Able to look after personal toilet and higiene unaided \\
6. Able to communicate with family or friends \\
7. Getting support from hospital doctors and nurses \\
8. Able to return to work or usual home activities \\
9. Feeling comfortable and in control \\
10.Having a feeling of general well-being \\
PART B: \\
Have you had any of the following in the last 24 hours? \\
11.Moderate pain \\
12.Severe pain \\
13.Nausea or vomiting \\
14.Feeling worried or anxious \\
15.Feeling sad or depressed
\end{tabular}

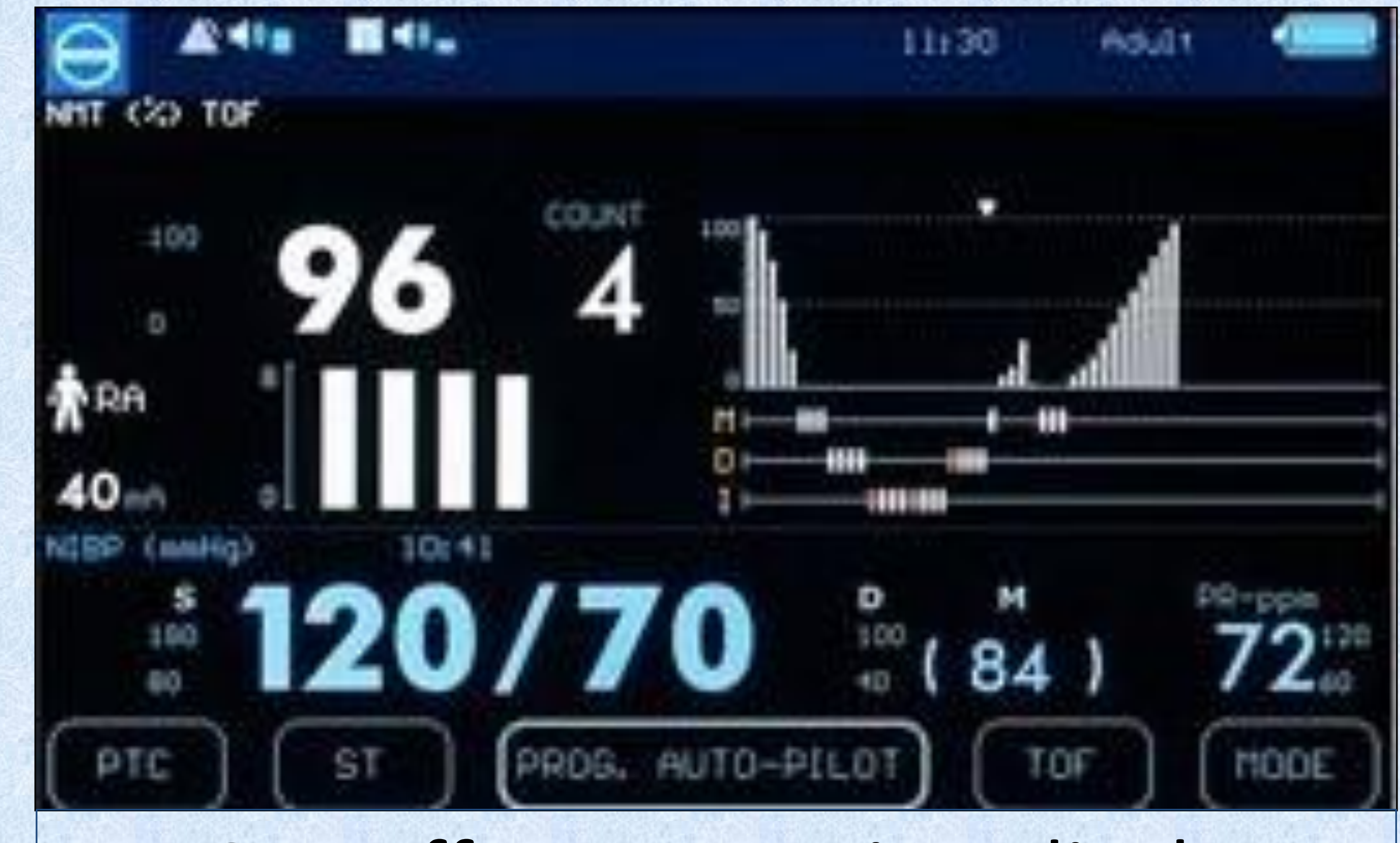

TOF-cuff NMT monitor display

\section{Results}

Five patients had an unpredicted admission, all of them due to surgical complications. Median pain score in PACU was 3.5 (interquartile range of 2 to 5); and 2 (interquartile range of 1 to 3 ) at $24 \mathrm{~h}$ after surgery. Six patients (8\%) presented mild nausea in PACU and none at $24 \mathrm{~h}$. The overall QoR-15 data decreased moderately at $24 \mathrm{~h}$ after surgery (Preoperative: median of 149 and interquartile range of 146-150; postoperative: median of 141 and interquartile range of $132-146) ; P<0.0001$. The percentage of decrease in QoR-15 in relation to baseline values was $7 \%$. The most affected parameters were in the emotional component, physical comfort and the presence of mild pain.

\section{Conclusion}

The application of a deep NMB in patients undergoing ambulatory LC provided an excellent postdischarge quality of recovery, with minimal pain and nausea in the early postoperative period. 